\title{
Note on Whittaker's Solution of Laplace's Equation.
}

\author{
By E. T. Copson.
}

(Received 1st February 1926. Read 5th February 1926.)

$\$ 1$. Whittaker * has shewn that a general solution of Laplace's Equation

$$
\frac{\hat{c}^{2} V}{\partial x^{2}}+\frac{\hat{\sigma}^{2} V}{\partial y^{2}}+\frac{\partial^{2} V}{\partial z^{2}}=0
$$

may be put in the form

$$
\int_{0}^{2 \pi} f(z+i x \cos u+i y \sin u, u) d u,
$$

where $f(v, u)$ denotes an arbitrary function of the two variables $u$ and $v$; such a representation is valid only in the neighbourhood of a regular point.

On account of the symmetry of the equation of Laplace, there are two other types of general solution, which are of the forms

$$
\begin{aligned}
& \int_{0}^{2 \pi} g(x+i y \cos u+i z \sin u, u) d u \\
& \int_{0}^{2 \pi} h(y+i z \cos u+i x \sin u, u) d u
\end{aligned}
$$

A given solution of Laplace's equation may be representable in all three of these forms; but each form is ralid in a different region of space, and is not transformable into either of the other forms. The simplest example of this is the solution

$$
2 \pi /\left\{x^{2}+y^{2}+z^{2}\right\}^{\frac{1}{2}}
$$

it has the definite integral representation

$$
\int_{0}^{2 \pi}(z+i x \cos u+i y \sin u)^{-1} d u
$$

which is only valid when $z>0$; when $z<0$, the integral represents $-2 \pi /\left\{x^{2}+y^{2}+z^{2}\right\}$; when $z=0$, the integral vanishes. There are two other representations valid when $x>0, y>0$ respectively.

*Muth. Ann. 57, (1902), 333. 
The representation $(1.4)$ is seen to be discontinuous at the plane $z=0$, which passes through the singular point $(0,0,0)$ of the solution.

In view of these facts, it seemed desirable to investigate whether the second solution of degree $(-1)$, viz. $Q_{0}(z / r) / r$, possesses definite integral representations, and to examine the connexion between the singular line $x=0, y=0$ of this solution with the region of validity of such integral representations.

$\S 2$. When we take polar coordinates, defined by

$$
x=r \sin \theta \cos \phi, y=r \sin \theta \sin \phi, z=r \cos \phi,
$$

the second solution of degree $(-1)$ has the form

$$
r^{-1} Q_{0}(\cos \theta)
$$

$\theta=0$ is the singular line.

Instead of this solution, we take the solution which has $\theta=\alpha, \phi=0$, as singular line, and then intend to examine what happens as $\alpha$ tends to zero. The solution is

where

$$
r^{-1} Q_{0}(\cos \varpi)
$$

$$
\cos \pi=\cos \theta \cos \alpha+\sin \theta \sin \alpha \cos \phi .
$$

Using the addition theorem for Legendre functions, ${ }^{*}$ we bave $r^{-1} Q_{0}(\cos \varpi)$

$$
=r^{-1} P_{0}(\cos \theta) Q_{0}(\cos \alpha)+2 \underset{m=1}{2} \sum^{-1} P_{0}{ }^{-m}(\cos \theta) \cos m \phi Q_{0}{ }^{m}(\cos \alpha) \ldots
$$

In this way, we have expressed our solution as an infinite series of terms which are expressible in the form $(1 \cdot 1)$. But this representation is only valid in a region for which

$$
\theta<\alpha \leq \pi / 2,
$$

i.e. inside a circular cone with $O z$ as axis and the singular line $\theta=\alpha, \phi=0$ as generator.

* See Whitriker and Watson: Modern Analyais (3rd Edn.) 329. We are using Hobson's definition (Phil. Trans. A 187 (1896)) of the associated functions. 
Now Hobson * has shewn that

$2 \pi P_{0}^{-m}(\cos \theta)(-1)^{m} \cos m \phi=\frac{1}{m !} \int_{0}^{2 \pi} \frac{\cos m u d u}{\cos \theta+i \sin \theta \cos (u-\phi)} \ldots$

Hence, if $z>0$, and if $\theta<\alpha \leq \pi / 2$, we have

$$
\begin{aligned}
2 \pi r^{-1} Q_{0}(\cos \varpi) & =Q_{0}(\cos \alpha) \int_{0}^{2 \pi} \frac{d u}{z+i x \cos u+i y \sin u} \\
& +2 \sum_{m=1}^{\infty} \frac{(-1)^{m}}{m !} Q_{0}{ }^{m}(\cos \alpha) \int_{0}^{2 \pi} \frac{\cos m u d u}{z+i x \cos u+i y \sin u} \ldots
\end{aligned}
$$

Before we invert the order of integration and summation, it is necessary to examine the uniformity of convergence of the series

$$
Q_{0}(\cos \alpha)+2 \sum_{m=1}^{\infty} \frac{(-1)^{m}}{m !} Q_{0}{ }^{m}(\cos \alpha) \cos m u
$$

It follows from some recent work $\dagger$ on the asymptotic expansions of the Hypergeometric Function that

$$
2 \frac{(-1)^{m}}{m !} Q_{0}^{m}(\cos \alpha) \sim \frac{i^{-m}}{m}\left[\cot ^{m} \frac{\alpha}{2}-(-1)^{m} \tan ^{m} \frac{\alpha}{2}+O\left(\frac{1}{m}\right)\right]
$$

for large values of $m$. The coefficients of the trigonometric series $(2 \cdot 4)$ are then not bounded as $m \rightarrow \infty$, unless $\alpha=\pi / 2$. If $\alpha=\pi / 2$, the series $(2 \cdot 4)$ converges uniformly in the interval $(0,2 \pi)$ except at $u=\pi / 2$ and $u=3 \pi / 2$, where it has finite discontinuities.

It is then legitimate to invert the order of integration and summation in $(2 \cdot 3)$, only if $\alpha=\pi / 2$. We have then

$$
2 \pi r^{-1} Q_{0}(\sin \theta \cos \phi)=\int_{0}^{2 \pi} \frac{f(u) d u}{z+i x \cos u+i y \sin u}
$$

where $f(u)$ is the sum of the series

$$
Q_{0}(0)+2 \sum_{m=1}^{\infty} \frac{(-1)}{m !} Q_{0}{ }^{m}(0) \cos m u,
$$

provided only that $z>0$.

Hence it is impossible to represent $Q_{0}(z / r) / r$ in the form $(1 \cdot 1)$. The solution $Q_{0}(x / r) / r$ has a definite integral representation of the

* Loc. cit., 499.

† G. N. Watsox: Camb. Phil. Trans., 22, (1918), 27i-308. 
form $(1 \cdot 1)$ valid when $z>0$, and, by a similar argument, a representation of the form $(1 \cdot 3)$ valid when $y>0$; in the former case, the integral represents $-Q_{0}(x / r) / r$ when $z<0$, and also in the latter when $y<0$

§3. We may write these conclusions thus:-

The solution $\left(x^{2}+y^{2}+z^{2}\right)^{-\frac{1}{2}} Q_{0}\left(z /\left(x^{2}+y^{2}+z^{2}\right)^{\frac{1}{2}}\right)$ cannot be represented as

$$
\int_{0}^{2 \pi} f(z+i x \cos u+i y \sin u, u) d u
$$

it can be represented in the forms

$$
\int_{0}^{2 \pi} g(x+i y \cos u+i z \sin u, u) d u
$$

and

$$
\int_{0}^{2 \pi} h(y+i z \cos u+i x \sin u, u) d u
$$

The forms (1-2) and (1.3) have different regions of validity, in each case bounded by a plane through the singular line $x=0, y=0$. 